\title{
Ground state solutions for Hamiltonian elliptic systems with super or asymptotically quadratic nonlinearity
}

\author{
Yubo He ${ }^{1}$, Dongdong Qin ${ }^{2}$ and Dongdong Chen ${ }^{2 *}$
}

"Correspondence:

cdd0525@163.com

${ }^{2}$ School of Mathematics and

Statistics, Central South University, Changsha, P.R. China

Full list of author information is

available at the end of the article

\section{Abstract}

This article concerns the Hamiltonian elliptic system:

$$
\begin{cases}-\Delta \varphi+V(x) \varphi=G_{\psi}(x, \varphi, \psi) & \text { in } \mathbb{R}^{N} \\ -\Delta \psi+V(x) \psi=G_{\varphi}(x, \varphi, \psi) & \text { in } \mathbb{R}^{N} \\ \varphi, \psi \in H^{1}\left(\mathbb{R}^{N}\right) . & \end{cases}
$$

Assuming that the potential $V$ is periodic and 0 lies in a spectral gap of $\sigma(-\Delta+V)$, least energy solution of the system is obtained for the super-quadratic case with a new technical condition, and the existence of ground state solutions of Nehari-Pankov type is established for the asymptotically quadratic case. The results obtained in the paper generalize and improve related ones in the literature.

MSC: $35 J 50 ; 35 J 55$

Keywords: Hamiltonian elliptic system; Super-quadratic; Asymptotically quadratic; Ground states; Strongly indefinite functionals

\section{Introduction and main results}

Consider the following Hamiltonian elliptic system:

$$
\begin{cases}-\Delta \varphi+V(x) \varphi=G_{\psi}(x, \varphi, \psi) & \text { in } \mathbb{R}^{N} \\ -\Delta \psi+V(x) \psi=G_{\varphi}(x, \varphi, \psi) & \text { in } \mathbb{R}^{N}, \\ \varphi, \psi \in H^{1}\left(\mathbb{R}^{N}\right), & \end{cases}
$$

where $\varphi, \psi: \mathbb{R}^{N} \rightarrow \mathbb{R}, V \in C\left(\mathbb{R}^{N}, \mathbb{R}\right)$, and $G \in C^{1}\left(\mathbb{R}^{N} \times \mathbb{R}^{2}, \mathbb{R}\right)$ with gradient $\nabla G=$ $\left(G_{\varphi}, G_{\psi}\right)$. Problem (1.1) or similar to (1.1) has been extensively investigated in the literature based on various assumptions on the potential $V(x)$ and the nonlinearity $G(x, \eta)$ with $\eta=(\varphi, \psi)$.

For the case of a bounded domain, assuming moreover $V(x) \equiv 0$, systems like or similar to (1.1) have been studied by some authors, see [7] for sublinear systems, $[8,9,17]$ for the superlinear case, [18] for asymptotically linear systems, and [28] for a singularly perturbed

(c) The Author(s) 2019. This article is distributed under the terms of the Creative Commons Attribution 4.0 International License (http://creativecommons.org/licenses/by/4.0/), which permits unrestricted use, distribution, and reproduction in any medium, provided you give appropriate credit to the original author(s) and the source, provide a link to the Creative Commons license, and indicate if changes were made. 
problem. Recently, an Orlitz space approach was used by De Figueiredo et al. [11] dealing the system with $G(x, \varphi, \psi)=F(\varphi)+H(\psi)$.

In [44], the authors studied ground state solutions for a Hamiltonian elliptic system with inverse square potential, and there are other papers concerned with the system in the whole space $\mathbb{R}^{N}$, see $[10,21-23,31,35,38-40,45-48]$ and the references therein. The main difficulty of such a problem is lack of the compactness of Sobolev embedding. The usual way to overcome this difficulty is working on the radially symmetric function space, which possesses compact embedding. Using spectral family theory of non-compact operator, De Figueiredo and Yang [10] proved the existence of a positive radially symmetric solution which decays exponentially to 0 at infinity for the case that $V \equiv 1$, see also [39] for the case that $G(x, \varphi, \psi)=F(x, \varphi)+H(x, \psi)$ is asymptotically autonomous. Later, this result was generalized by Sirakov [31] in a different way. Infinitely many radial as well as non-radial solutions were obtained by Bartsch and De Figueiredo [4] provided that $G(x, \eta)$ is even in $\eta$. In [21], Li and Yang proved, via a generalized linking theorem, that (1.1) has a positive ground state solution with $V \equiv 1$ and asymptotically autonomous quadratic nonlinearity, see also [48] for a super-quadratic case. The case of general periodic potential was considered in $[38,47]$ for super-quadratic systems with $G(x, \varphi, \psi)=F(x, \varphi)+H(x, \psi)$ and $[45,46]$ for asymptotically quadratic systems. Assuming that $V$ is periodic and positive, nontrivial solutions were obtained by Liao et al. in the recent paper [22] under some new super-quadratic conditions. The nonperiodic and asymptotically quadratic case was studied in [36, 41, 42]. For semiclassical problems with subcritical and critical nonlinearities, we refer to $[14,22]$.

Here, we mention the dual variational method, another usual way to avoid the indefinite character of the original functional. See, for instance, Ávila and Yang [2, 3], Alves et al. [1], Yang [39], and the references therein.

As pointed out in $[40,46,47]$, besides the lack of the compactness of Sobolev embedding, there are two kinds of indefiniteness we have to face: one comes from the system itself and the other comes from each equation in the system. Although a proper variational setting for (1.1) can be established, the functional associated with it is strongly indefinite. Therefore the periodic assumptions on $V$ and $G$ are usually needed, see, for instance, [19, 33-35, 40, 43, 45-47]. Applying the critical point theory developed by Bartsch and Ding [5], Zhao et al. [46] obtained a least energy solution for asymptotically quadratic system (1.1), i.e., a nontrivial solution $\bar{\eta} \in E$ satisfying $I(\bar{\eta})=\inf _{\mathcal{M}} I$, where

$$
\mathcal{M}:=\left\{\eta \in E \backslash\{(0,0)\}: I^{\prime}(\eta)=0\right\},
$$

$E=E^{-} \oplus E^{+}$is the working space on which the energy functional $I$ associated with (1.1) is defined, see (2.12). For the super-quadratic case, a least energy solution of (1.1) was also established by Zhang et al. [40] via the critical point theory constructed in [5]. Based on the work [34], Tang [35] developed a direct approach and found a ground state solution of Nehari-Pankov type for (1.1), i.e., a nontrivial solution $\bar{\eta} \in E$ satisfying $I(\bar{\eta})=\inf _{\mathcal{N}^{-}} I$, where

$$
\mathcal{N}^{-}=\left\{\eta \in E \backslash E^{-}:\left\langle I^{\prime}(\eta), \eta\right\rangle=\left\langle I^{\prime}(\eta), w\right\rangle=0, \forall w \in E^{-}\right\}
$$

The main ingredients in this approach are the observations that, for any $\eta \in E \backslash E^{-}$, there exist $t=t(\eta)>0$ and $w=w(\eta) \in E^{-}$such that $w+t \eta \in \mathcal{N}^{-}$; moreover, a minimizing Ce- 
rami sequence for the energy functional $I$ can be found outside $\mathcal{N}^{-}$by using the diagonal method. The set $\mathcal{N}^{-}$first introduced by Pankov [27] is a subset of the Nehari manifold

$$
\mathcal{N}=\left\{\eta \in E \backslash\{(0,0)\}:\left\langle I^{\prime}(\eta), \eta\right\rangle=0\right\} .
$$

Note that, if $\eta \neq(0,0)$ and $I^{\prime}(\eta)=0$, then $\eta \in \mathcal{N}^{-}$. Hence $\mathcal{N}^{-}$contains all nontrivial critical points of $I$, i.e., $\mathcal{M}$ is a very small subset of $\mathcal{N}^{-}$. In general, it is more difficult to find a solution $\bar{\eta}$ for (1.1) that satisfies $I(\bar{\eta})=\inf _{\mathcal{N}^{-}} I$ than the one satisfying $I(\bar{\eta})=\inf _{\mathcal{M}} I$.

Let

$$
\Lambda:=\max \{-\underline{\Lambda}, \bar{\Lambda}\}, \quad \Lambda_{0}=\min \{-\underline{\Lambda}, \bar{\Lambda}\} .
$$

It follows from (1.6) that $\Lambda_{0}>0$. The following assumptions were introduced in [40, 46] for super and asymptotically quadratic system (1.1):

(V) $V \in C\left(\mathbb{R}^{N}\right)$ are 1-periodic in $x_{i}, i=1,2, \ldots, N$, and 0 lies in a gap of the spectrum of $-\Delta+V$, i.e.,

$$
\sup [\sigma(-\triangle+V) \cap(-\infty, 0)]:=\underline{\Lambda}<0<\bar{\Lambda}:=\inf [\sigma(-\triangle+V) \cap(0, \infty)]
$$

(G1) $G \in C^{1}\left(\mathbb{R}^{N} \times \mathbb{R}^{2},[0, \infty)\right)$ is 1-periodic in $x_{i}, i=1,2, \ldots, N$, and $\left|G_{\eta}(x, \eta)\right|=o(|\eta|)$ as $|\eta| \rightarrow 0$ uniformly in $x$, where $G_{\eta}(x, \eta)=\nabla G(x, \eta)$;

(SQ) $\lim _{|\eta| \rightarrow \infty} \frac{G(x, \eta)}{|\eta|^{2}}=\infty$ uniformly in $x \in \mathbb{R}^{N}$;

(DL) $\hat{G}(x, \eta):=\frac{1}{2} G_{\eta}(x, \eta) \cdot \eta-G(x, \eta)>0$ if $\eta \neq(0,0)$, and there exist $r_{0}>0, c_{0}>0$, and $\sigma>\max \{1, N / 2\}$ such that

$$
\left|G_{\eta}(x, \eta)\right|^{\sigma} \leq c_{0} \hat{G}(x, \eta)|\eta|^{\sigma}, \quad \forall(x, \eta) \in \mathbb{R}^{N} \times \mathbb{R}^{2},|\eta| \geq r_{0},
$$

where and in the sequel, the dot denotes the inner product in $\mathbb{R}^{2}$;

$\left(G_{2}\right) G_{\eta}(x, \eta)=V_{\infty}(x) \eta+\bar{G}_{\eta}(x, \eta)$ with $\bar{G}_{\eta}(x, \eta)=\nabla \bar{G}(x, \eta)$, where $V_{\infty} \in C\left(\mathbb{R}^{N}\right)$ is 1periodic in each of $x_{1}, x_{2}, \ldots, x_{N}$, inf $V_{\infty}>\Lambda$, and $\left|\bar{G}_{\eta}(x, \eta)\right|=o(|\eta|)$ as $|\eta| \rightarrow \infty$ uniformly in $x \in \mathbb{R}^{N}$;

$\left(\mathrm{G}_{3}\right) \hat{G}(x, \eta)>0$ if $\eta \neq(0,0)$, and $\hat{G}(x, \eta) \rightarrow \infty$ as $|\eta| \rightarrow \infty$;

$\left(\mathrm{G}_{4}\right) \hat{G}(x, \eta) \geq 0$, and there exist $\delta_{0} \in\left(0, \Lambda_{0}\right), \delta_{1}>0$ such that $\hat{G}(x, \eta)>0$ if $0<|\eta| \leq \delta_{1}$, and $\hat{G}(x, \eta) \geq \delta_{0}$ whenever $\left|\hat{G}_{\eta}(x, \eta)\right| \geq\left(\Lambda_{0}-\delta_{0}\right)|\eta|$.

More precisely, the following theorems were established in $[40,46]$.

Theorem 1.1 ([40]) Let (V), (G1), (SQ), and (DL) be satisfied. Then (1.1) has a least energy solution, i.e., a nontrivial solution $\bar{\eta} \in E$ such that $I(\bar{\eta})=\inf _{\mathcal{M}} I$.

Theorem $1.2([46])$ Let $(\mathrm{V}),(\mathrm{G} 1),\left(\mathrm{G}_{2}\right)$, and $\left(\mathrm{G}_{3}\right)$ or $\left(\mathrm{G}_{4}\right)$ be satisfied. Then (1.1) has a least energy solution.

It follows from (G1), (SQ), and (DL) that the following condition holds, see Lemma 2.2:

(G0) $G \in C^{1}\left(\mathbb{R}^{N} \times \mathbb{R}^{2}, \mathbb{R}\right)$, and there exist constants $p \in\left(2,2^{*}\right)$ and $C_{1}>0$ such that

$$
\left|G_{\eta}(x, \eta)\right| \leq C_{1}\left(1+|\eta|^{p-1}\right), \quad \forall(x, \eta) \in \mathbb{R}^{N} \times \mathbb{R}^{2},
$$

where $2^{*}:=2 N /(N-2)$ if $N \geq 3$ and $2^{*}:=+\infty$ if $N=1$ or 2 . 
Condition (DL) was first introduced by Ding and Lee [13] for a single Schrödinger equation, and it together with (SQ) is commonly used instead of the following classic condition, see, for instance, [26, 40, 42].

(AR) There exists a constant $\mu>2$ such that

$$
0<\mu G(x, \eta) \leq G_{\eta}(x, \eta) \cdot \eta, \quad \forall x \in \mathbb{R}^{N}, \eta \in \mathbb{R}^{2} \backslash\{(0,0)\}
$$

It is clear that (AR) implies (SQ); moreover, by a similar argument as in the proof of [13, Lemma 1.3], (DL) holds provided that (AR) and (G0) with $\mu \geq p$ are satisfied. The idea of using the more natural super-quadratic condition (SQ) under a Nehari type setting to replace (AR) goes back to Liu and Wang [25]. Afterwards, condition (SQ) was used in many papers, see $[13,14,26,33,38,42,47]$ and the references therein. In a recent paper [30], a local superquadratic condition instead of (SQ) was introduced by Qin, Tang, and $\mathrm{Wu}$, which combines with a technical condition to guarantee the existence of ground state solutions for Schrödinger systems like (1.1). Note that the assumption $\hat{G}(x, \eta)>0$ in (DL), $\left(G_{3}\right)$, and $\left(G_{4}\right)$ plays an important role in the arguments of Zhang [40] and Zhao [46], see also Ding and Lee [13].

Inspired by the aforementioned works, we continue to study problem (1.1) in this paper and construct two types of ground state solutions, i.e., the least energy solution and the Nehari-Pankov type. We first use a technical condition introduced in [29] to consider the super-quadratic case and obtain a least energy solution for (1.1) with the aid of a generalized linking theorem established in [20]. Then we lay emphasis on the asymptotically quadratic case and discuss the existence of ground state solution of Nehari-Pankov type for (1.1). Different from the super-quadratic case, the Nehari-Pankov manifold $\mathcal{N}^{-}$is not homeomorphic to the unit sphere for the asymptotically quadratic case, so the generalized Nehari manifold method introduced by Szulkin and Weth [33] does not apply even under strict monotonicity on $G$. More precisely, for each $\eta \in E \backslash E^{-}, \mathcal{N}^{-}$does not necessarily intersect the set $E^{-} \oplus \mathbb{R}^{+} \eta$, so one cannot directly adopt the method used in [35] either. On the other hand, the argument in [40,46, see Lemma 4.1] becomes invalid due to the lack of positive assumption on $\hat{G}(x, \eta)$ (i.e., $\hat{G}(x, \eta)>0$ ). Motivated by the works [27, $33,40,46]$, we further develop the approach in $[29,34,35]$ to find ground state solution of Nehari-Pankov type for (1.1). Our approach is based on finding a proper subset $E_{0}^{+}$of $E \backslash E^{-}$(see (3.4) for the definition) such that, for any $\eta \in E_{0}^{+}$, there exist $t=t(\eta)>0$ and $w=w(\eta) \in E^{-}$satisfying $w+t \eta \in \mathcal{N}^{-}$, then we can derive a minimizing sequence on the Nehari-Pankov manifold by using the diagonal method, see Lemma 3.9.

Before stating our main results, we first introduce the following weaker version of (DL):

(G2) $\hat{G}(x, \eta) \geq 0$, and there exist $C_{0}>0, \delta_{0} \in\left(0, \Lambda_{0}\right)$, and $\sigma>\max \{1, N / 2\}$ such that

$$
\frac{\left|G_{\eta}(x, \eta)\right|}{|\eta|} \geq \frac{1}{2}\left(\Lambda_{0}-\delta_{0}\right) \quad \Longrightarrow \quad\left|G_{\eta}(x, \eta)\right|^{\sigma} \leq C_{0} \hat{G}(x, \eta)|\eta|^{\sigma} .
$$

Clearly, (G2) holds under (DL) and (G1). Thus (G2) weakens (DL), and there are some functions satisfying (G2), but not (DL) and (AR), see Examples 1.9 and 1.10. Moreover, (G0) holds under (G1), (G2), and (SQ), see Lemma 2.2.

We are now in a position to state the first result of this paper.

Theorem 1.3 Let (V), (G1), (G2), and (SQ) be satisfied. Then (1.1) has a least energy solution. 
Next, we consider the asymptotically quadratic case and introduce the following assumptions:

(G3) $G_{\eta}(x, \eta)=V_{\infty}(x) \eta+\bar{G}_{\eta}(x, \eta)$, where $V_{\infty} \in C\left(\mathbb{R}^{N}\right)$ is 1-periodic in each of $x_{1}, x_{2}, \ldots$, $x_{N}$ with $\inf V_{\infty}>0$, and there exists $\eta_{0} \in E^{+} \backslash\{0\}$ such that

$$
\left\|\eta_{0}\right\|^{2}-\|w\|^{2}-\int_{\mathbb{R}^{N}} V_{\infty}(x)\left|\eta_{0}+w\right|^{2} \mathrm{~d} x<0, \quad \forall w \in E^{-}
$$

$G_{\eta}(x, \eta) \cdot \bar{G}_{\eta}(x, \eta) \leq 0$ and $G_{\eta}(x, \eta) \cdot \bar{G}_{\eta}(x, \eta)<0$ for $0<|\eta| \leq \alpha_{0}$ for some $\alpha_{0}>0$, $\left|\bar{G}_{\eta}(x, \eta)\right|=o(|\eta|)$ as $|\eta| \rightarrow \infty$ uniformly in $x \in \mathbb{R}^{N}$;

(G3') $G_{\eta}(x, \eta)=V_{\infty}(x) \eta+\bar{G}_{\eta}(x, \eta)$, where $V_{\infty} \in C\left(\mathbb{R}^{N}\right)$ is 1-periodic in each of $x_{1}, x_{2}, \ldots$, $x_{N}$ with $\inf V_{\infty}>\Lambda,\left|\bar{G}_{\eta}(x, \eta)\right|=o(|\eta|)$ as $|\eta| \rightarrow \infty$ uniformly in $x \in \mathbb{R}^{N}$, and $G_{\eta}(x, \eta) \cdot \bar{G}_{\eta}(x, \eta)<0$ for $x \in \mathbb{R}^{N}$ and $\eta \neq(0,0) ;$

(G4) For all $\theta \geq 0, x \in \mathbb{R}^{N}, \eta, w \in \mathbb{R}^{2}$, there holds

$$
\frac{1-\theta^{2}}{2} G_{\eta}(x, \eta) \cdot \eta-\theta G_{\eta}(x, \eta) \cdot w+G(x, \theta \eta+w)-G(x, \eta) \geq 0
$$

Our main results for the asymptotically quadratic case read as follows.

Theorem 1.4 Let (V), (G1), (G3), and (G4) be satisfied. Then (1.1) has a nontrivial solution $\bar{\eta} \in E$ such that $I(\bar{\eta})=\inf _{\mathcal{N}^{-}} I \geq \kappa$, where $\kappa>0$ is a constant.

Corollary 1.5 Let (V), (G1), (G3'), and (G4) be satisfied. Then (1.1) has a nontrivial solution $\bar{\eta} \in E$ such that $I(\bar{\eta})=\inf _{\mathcal{N}^{-}} I \geq \kappa$, where $\kappa>0$ is a constant.

In general, $\mathcal{N}^{-}$contains infinitely many elements of $E$. In fact, for any $\eta \in E_{0}^{+}$defined later by (3.4), there exist $t=t(\eta)>0$ and $w=w(\eta) \in E^{-}$such that $w+t \eta \in \mathcal{N}^{-}$which is the global maximum of $\left.I\right|_{E^{-} \oplus \mathbb{R}^{+} \eta}$, see Corollary 3.2 and Lemma 3.7. Since $\bar{\eta}$ is a solution at which $I$ has least "energy" in set $\mathcal{N}^{-}$, it was called a ground state solution of Nehari-Pankov type in $[33,35]$.

Remark 1.6 It is easy to see that (G4), together with $\left|G_{\eta}(x, \eta)\right|=o(|\eta|)$ as $|\eta| \rightarrow 0$ uniformly in $x \in \mathbb{R}^{N}$, implies that $G(x, \eta) \geq 0$ and $\hat{G}(x, \eta) \geq 0$ for any $(x, \eta) \in \mathbb{R}^{N} \times \mathbb{R}^{2}$. Condition (G0) holds under (G1) and (G3); moreover, (G3') implies (G3), see (2.14) for details. The crucial condition $\hat{G}(x, \eta)>0$ in (DL), $\left(\mathrm{G}_{3}\right)$, and $\left(\mathrm{G}_{4}\right)$ is not needed in (G2) and (G4); moreover, there are many functions satisfying (G2), (G3), and (G4), but not (DL), (G3'), and (G4') resp., see Examples 1.10 and 1.11. Thus Theorems 1.3 and 1.4 generalize and improve the results in $[35,40,46]$.

Remark 1.7 In [35], Tang introduced the following class of functions which satisfies (G4):

$$
G(x, \varphi, \psi)=\sum_{i=1}^{k} \int_{0}^{\alpha_{i} \varphi+\beta_{i} \psi} g_{i}(x, t) t \mathrm{~d} t+\sum_{j=1}^{l} \int_{0}^{\sqrt{\varphi^{2}+2 b_{j} \varphi \psi+a_{j} \psi^{2}}} h_{j}(x, t) t \mathrm{~d} t
$$

where $\alpha_{i}, \beta_{i}, a_{j}, b_{j} \in \mathbb{R}$ with $\alpha_{i}^{2}+\beta_{i}^{2} \neq 0$ and $a_{j}>b_{j}^{2}, g_{i}(x, t)$ and $h_{j}(x, t)$ are nondecreasing in $t \in \mathbb{R}^{+}$for every $x \in \mathbb{R}^{N}$ and $g_{i}(x, 0)=h_{j}(x, 0)=0$. 
Condition (G4) is also satisfied under assumptions (G1), $\left(\mathrm{G}_{2}\right)$ and the following condition introduced by Bartsch and Mederski [6], the proof of which will be given in the Appendix.

(G4') $\hat{G}(x, \eta) \geq 0$, and for any $x \in \mathbb{R}^{N}, \eta, w \in \mathbb{R}^{2}$, there holds

$$
2 G_{\eta}(x, \eta) \cdot \eta[G(x, \eta)-G(x, w)] \leq\left(G_{\eta}(x, \eta) \cdot \eta\right)^{2}-\left(G_{\eta}(x, \eta) \cdot w\right)^{2},
$$

whenever $G_{\eta}(x, \eta) \cdot w=G_{\eta}(x, w) \cdot \eta \neq 0$.

Moreover, (G4) holds also under conditions (G1), (G4'), and (SQ).

Remark 1.8 For the asymptotically quadratic case, Nehari-Pankov manifold $\mathcal{N}^{-}$does not necessarily intersect the set $\hat{E}(\eta):=E^{-} \oplus \mathbb{R}^{+} \eta$ for each $\eta \in E \backslash E^{-}$, and so $\mathcal{N}^{-}$is not homeomorphic to the unit sphere $S^{+} \subset E^{+}$even under the strict monotonic condition. Thus, it seems infeasible to find a minimizing sequence on the Nehari-Pankov manifold by reducing the problem on a sphere under assumptions (G1), (G3), and (G4).

Before proceeding to the proof of main results, we give some nonlinear examples to illustrate condition (G2).

Example $1.9 G(x, \eta)=\alpha(x)|\eta|^{2} \ln [1+|\eta|]$, where $\alpha \in C\left(\mathbb{R}^{N},(0, \infty)\right)$ is 1-periodic in $x_{i}, i=$ $1,2, \ldots, N$.

It is not difficult to verify that $G$ satisfies (G1), (G2), and (SQ), but it does not satisfy (AR).

Example $1.10 G(x, \eta)=a\left(8 / 5|\eta|^{13 / 4}-4|\eta|^{11 / 4}+9 / 2|\eta|^{9 / 4}\right)$, where $a>0$ and $N \leq 4$.

By simple computation, one has $\hat{G}(x, \eta)=a|\eta|^{9 / 4}(\sqrt{|\eta|}-3 / 4)^{2} \geq 0$. Then $G$ does not satisfy (AR) and (DL), but it satisfies (G2) with $\sigma=12 / 5$ if $a \in\left(0,8 \Lambda_{0} / 81\right.$ ).

For the asymptotically quadratic case, it is not difficult to verify that the following functions satisfy (G1), (G3), (G4), or (G3') by virtue of Remark 1.7, but Example 1.11 does not satisfy $\left(\mathrm{G}_{3}\right)$ and $\left(\mathrm{G}_{4}\right)$ since $\hat{G}(x, \eta)=0$ if $|\eta| \geq 1$.

Example $1.11 G_{\eta}(x, \eta)=V_{\infty}(x) \max \left\{|\eta|^{\alpha}, 1\right\} \eta$, where $\alpha>0$ and $V_{\infty} \in C\left(\mathbb{R}^{N}\right)$ is 1-periodic in each of $x_{1}, x_{2}, \ldots, x_{N}$ with inf $V_{\infty}>\Lambda$.

Example $1.12 G_{\eta}(x, \eta)=V_{\infty}(x)\left[1-\frac{1}{1+|\eta|^{\alpha}}\right] \eta$, where $\alpha>0$ and $V_{\infty} \in C\left(\mathbb{R}^{N}\right)$ is 1 -periodic in each of $x_{1}, x_{2}, \ldots, x_{N}$ with $\inf V_{\infty}>\Lambda$.

Example $1.13 G_{\eta}(x, \eta)=V_{\infty}(x)\left[1-\frac{1}{\ln (e+|\eta|)}\right] \eta$, where $V_{\infty} \in C\left(\mathbb{R}^{N}\right)$ is 1-periodic in each of $x_{1}, x_{2}, \ldots, x_{N}$ with $\inf V_{\infty}>\Lambda$.

The remainder of this paper is organized as follows. In Sect. 2, some preliminary results are presented. The proofs of the main results will be given in the last section.

\section{Variational setting and preliminaries}

Let $\mathcal{A}=-\triangle+V$. Then $\mathcal{A}$ is self-adjoint in $L^{2}\left(\mathbb{R}^{N}\right)$ with domain $\mathfrak{D}(\mathcal{A})=H^{2}\left(\mathbb{R}^{N}\right)$ (see $[16$, Theorem 4.26]). Let $\{\mathcal{E}(\lambda):-\infty \leq \lambda \leq+\infty\}$ and $|\mathcal{A}|$ be the spectral family and the absolute 
value of $\mathcal{A}$, respectively, and $|\mathcal{A}|^{1 / 2}$ be the square root of $|\mathcal{A}|$. Set $\mathcal{U}=\mathrm{id}-\mathcal{E}(0)-\mathcal{E}(0-)$. Then $\mathcal{U}$ commutes with $\mathcal{A},|\mathcal{A}|$, and $|\mathcal{A}|^{1 / 2}$, and $\mathcal{A}=\mathcal{U}|\mathcal{A}|$ is the polar decomposition of $\mathcal{A}$ (see [15, Theorem IV 3.3]). Let

$$
H:=\mathfrak{D}\left(|\mathcal{A}|^{1 / 2}\right), \quad H^{-}:=\mathcal{E}(0) H, \quad H^{+}:=[\mathrm{id}-\mathcal{E}(0)] H .
$$

For any $u \in H$, it is easy to see that

$$
u=u^{-}+u^{+}, \quad u^{-}:=\mathcal{E}(0) u \in H^{-}, \quad u^{+}:=[\mathrm{id}-\mathcal{E}(0)] u \in H^{+}
$$

and

$$
\mathcal{A} u^{-}=-|\mathcal{A}| u^{-}, \quad \mathcal{A} u^{+}=|\mathcal{A}| u^{+}, \quad \forall u \in H \cap \mathfrak{D}(\mathcal{A}) .
$$

Define an inner product

$$
(u, v)_{H}=\left(|\mathcal{A}|^{1 / 2} u,|\mathcal{A}|^{1 / 2} v\right)_{L^{2}}, \quad u, v \in H
$$

and the corresponding norm

$$
\|u\|_{H}=\left\||\mathcal{A}|^{1 / 2} u\right\|_{L^{2}}, \quad u \in H
$$

where $(\cdot, \cdot)_{L^{2}}$ denotes the inner product of $L^{2}\left(\mathbb{R}^{N}\right),\|\cdot\|_{L^{s}}$ stands for the usual $L^{s}\left(\mathbb{R}^{N}\right)$ norm. By (V), $H=H^{1}\left(\mathbb{R}^{N}\right)$ with equivalent norms. Therefore, $H$ embeds continuously in $L^{s}\left(\mathbb{R}^{N}\right)$ for all $2 \leq s \leq 2^{*}$. In addition, one has the decomposition $H=H^{-} \oplus H^{+}$which are orthogonal with respect to $(\cdot, \cdot)_{L^{2}}$ and $(\cdot, \cdot)_{H}$. Then there hold

$$
\int_{\mathbb{R}^{N}}\left(|\nabla u|^{2}+V(x)|u|^{2}\right) \mathrm{d} x=\left\|u^{+}\right\|_{H}^{2}-\left\|u^{-}\right\|_{H^{2}}^{2}, \quad \forall u=u^{-}+u^{+} \in H .
$$

Let $E=H \times H$ with the inner product

$$
((u, v),(\varphi, \psi))=(u, \varphi)_{H}+(v, \psi)_{H},
$$

and the corresponding norm

$$
\|z\|=\left[\|u\|_{H}^{2}+\|v\|_{H}^{2}\right]^{1 / 2}, \quad \forall z=(u, v) \in E .
$$

By (1.6), (1.5), and (2.8), it is easy to see that $\|z\|^{2} \geq \Lambda_{0}\|z\|_{2}^{2}$ for any $z \in E$, where $\|\cdot\|_{s}$ stands for the usual $L^{s}\left(\mathbb{R}^{N}, \mathbb{R}^{2}\right)$ norm. Moreover, we have the following lemma.

Lemma 2.1 Let $(\mathrm{V})$ be satisfied. Then the embedding $E \hookrightarrow L^{p}\left(\mathbb{R}^{N}, \mathbb{R}^{2}\right)$ is continuous for all $p \in\left[2,2^{*}\right]$ and $E \hookrightarrow L_{\mathrm{loc}}^{p}\left(\mathbb{R}^{N}, \mathbb{R}^{2}\right)$ is compact for all $p \in\left[2,2^{*}\right)$.

Lemma 2.2 ([29, Lemma 2.2]) Suppose that (G1), (G2), and (SQ) are satisfied. Then (G0) holds. Moreover, for any $\varepsilon>0$, there exist $C_{\varepsilon}>0$ and $p \in\left(2,2^{*}\right)$ such that

$$
\left|G_{\eta}(x, \eta)\right| \leq \varepsilon|\eta|+C_{\varepsilon}|\eta|^{p-1}, \quad \forall(x, \eta) \in \mathbb{R}^{N} \times \mathbb{R}^{2},
$$

and $\hat{G}(x, \eta) \rightarrow \infty$ as $|\eta| \rightarrow \infty$ uniformly in $x$. 
Under (V), (G0), and (G1), a standard argument (see [12, 32, 37]) shows that the solutions of problem (1.1) are critical points of the functional

$$
I(\eta)=\int_{\mathbb{R}^{N}}(\nabla \varphi \nabla \psi+V(x) \varphi \psi) \mathrm{d} x-\int_{\mathbb{R}^{N}} G(x, \eta) \mathrm{d} x, \quad \forall \eta=(\varphi, \psi) \in E,
$$

$I$ is of class $C^{1}(E, \mathbb{R})$, and

$$
\begin{aligned}
\left\langle I^{\prime}(\eta), w\right\rangle= & \int_{\mathbb{R}^{N}}(\nabla \varphi \nabla \phi+V(x) \varphi \phi) \mathrm{d} x+\int_{\mathbb{R}^{N}}(\nabla \psi \nabla \xi+V(x) \psi \xi) \mathrm{d} x \\
& -\int_{\mathbb{R}^{N}}\left(G_{\varphi}(x, \eta) \xi+G_{\psi}(x, \eta) \phi\right) \mathrm{d} x, \quad \forall \eta=(\varphi, \psi), w=(\xi, \phi) \in E .
\end{aligned}
$$

The following generalized linking theorem plays an important role in proving our main results.

Let $X$ be a Hilbert space with $X=X^{-} \oplus X^{+}$and $X^{-} \perp X^{+}$. For a functional $I \in C^{1}(X, \mathbb{R}), I$ is said to be weakly sequentially lower semi-continuous if, for any $u_{n} \rightarrow u$ in $X$, one has $I(u) \leq$ $\liminf _{n \rightarrow \infty} I\left(u_{n}\right)$, and $I^{\prime}$ is said to be weakly sequentially continuous if $\lim _{n \rightarrow \infty}\left\langle I^{\prime}\left(u_{n}\right), v\right\rangle=$ $\left\langle I^{\prime}(u), v\right\rangle$ for each $v \in X$.

Lemma 2.3 ([19], [20, Theorem 2.1]) Let $X$ be a Hilbert space with $X=X^{-} \oplus X^{+}$and $X^{-} \perp X^{+}$, and let $I \in C^{1}(X, \mathbb{R})$ of the form

$$
I(u)=\frac{1}{2}\left(\left\|u^{+}\right\|^{2}-\left\|u^{-}\right\|^{2}\right)-\phi(u), \quad u=u^{+}+u^{-} \in X^{+} \perp X^{-} .
$$

\section{Suppose that the following assumptions are satisfied:}

(I1) $\phi \in C^{1}(X, \mathbb{R})$ is bounded from below and weakly sequentially lower semi-continuous;

(I2) $\phi^{\prime}$ is weakly sequentially continuous;

(I3) there exist $r>\rho>0$ and $e \in X^{+}$with $\|e\|=1$ such that

$$
\kappa:=\inf I\left(S_{\rho}\right)>\sup I(\partial Q)
$$

where

$$
S_{\rho}=\left\{u \in X^{+}:\|u\|=\rho\right\}, \quad Q=\left\{s e+v: v \in X^{-}, s \geq 0,\|s e+v\| \leq r\right\}
$$

Then, for some $c \geq \kappa$, there exists a sequence $\left\{u_{n}\right\} \subset X$ satisfying

$$
I\left(u_{n}\right) \rightarrow c, \quad\left\|I^{\prime}\left(u_{n}\right)\right\|\left(1+\left\|u_{n}\right\|\right) \rightarrow 0
$$

Let

$$
E^{+}=H^{+} \times H^{-}, \quad E^{-}=H^{-} \times H^{+},
$$

then, for any $z=(u, v) \in E$, there holds

$$
z=z^{+}+z^{-}, \quad z^{+}=\left(u^{+}, v^{-}\right) \in E^{+}, \quad z^{-}=\left(u^{-}, v^{+}\right) \in E^{-} .
$$


Moreover, $E^{+}$and $E^{-}$are orthogonal with respect to the inner products $(\cdot, \cdot)_{2}$ and $(\cdot, \cdot)$, where $(\cdot, \cdot)_{2}$ is chosen by $((u, v),(\varphi, \psi))_{2}=(u, \varphi)_{L^{2}}+(v, \psi)_{L^{2}}$ for any $(u, v),(\varphi, \psi) \in$ $L^{2}\left(\mathbb{R}^{N}, \mathbb{R}^{2}\right)$. Hence $E=E^{+} \oplus E^{-}$.

Next we show that (G3') implies (G3). If inf $V_{\infty}>\Lambda$, take $\bar{\mu} \in\left(\Lambda, \inf V_{\infty}\right)$, then there exists $\eta_{0} \in(\mathcal{E}(\bar{\mu})-\mathcal{E}(0)) H \times(\mathcal{E}(0)-\mathcal{E}(-\bar{\mu})) H \subset E^{+} \backslash\{0\}$ such that $\Lambda\left\|\eta_{0}\right\|_{2}^{2} \leq\left\|\eta_{0}\right\|^{2} \leq \bar{\mu}\left\|\eta_{0}\right\|_{2}^{2}$, thus for any $w \in E^{-}$, one has

$$
\begin{aligned}
& \left\|\eta_{0}\right\|^{2}-\|w\|^{2}-\int_{\mathbb{R}^{N}} V_{\infty}(x)\left|\eta_{0}+w\right|^{2} \mathrm{~d} x \\
& \quad \leq \bar{\mu}\left\|\eta_{0}\right\|_{2}^{2}-\|w\|^{2}-\inf V_{\infty}\left(\left\|\eta_{0}\right\|_{2}^{2}+\|w\|_{2}^{2}\right) \\
& \quad=-\left[\left(\inf V_{\infty}-\bar{\mu}\right)\left\|\eta_{0}\right\|_{2}^{2}+\|w\|^{2}+\inf V_{\infty}\left(\left\|\eta_{0}\right\|_{2}^{2}+\|w\|_{2}^{2}\right)\right] \\
& \quad<0 .
\end{aligned}
$$

This shows that (1.7) holds.

As in [46], we introduce a change of variable

$$
\left\{\begin{array}{l}
\varphi=\frac{u+v}{\sqrt{2}}, \\
\psi=\frac{u-v}{\sqrt{2}},
\end{array}\right.
$$

and set $H(x, z)=H(x, u, v):=G\left(x, \frac{u+v}{\sqrt{2}}, \frac{u-v}{\sqrt{2}}\right), \bar{H}(x, z)=\bar{G}\left(x, \frac{u+v}{\sqrt{2}}, \frac{u-v}{\sqrt{2}}\right)$, here and in the sequel, we write $z=(u, v)$ and $|z|=\left(|u|^{2}+|v|^{2}\right)^{\frac{1}{2}}$.

It is not difficult to verify that assumptions (G2'), $\left(\mathrm{G}^{\prime}\right)$, and (G0)-(G5) on $G$ imply that $H$ satisfies:

(S0) There exist constants $p \in\left(2,2^{*}\right)$ and $C_{1}>0$ such that

$$
\left|H_{z}(x, z)\right| \leq C_{1}\left(1+|z|^{p-1}\right), \quad \forall(x, z) \in \mathbb{R}^{N} \times \mathbb{R}^{2}
$$

(S1) $H \in C^{1}\left(\mathbb{R}^{N} \times \mathbb{R}^{2},[0, \infty)\right)$ is 1-periodic in $x_{i}, i=1,2, \ldots, N$, and $\left|H_{z}(x, z)\right|=o(|z|)$ as $|z| \rightarrow 0$ uniformly in $x$;

(S2) $\hat{H}(x, z):=\frac{1}{2} H_{z}(x, z) \cdot z-H(x, z) \geq 0$, and there exist constants $C_{0}>0, \delta_{0} \in\left(0, \Lambda_{0}\right)$ and $\sigma>\max \{1, N / 2\}$ such that

$$
\frac{\left|H_{z}(x, z)\right|}{|z|} \geq \frac{1}{2}\left(\Lambda_{0}-\delta_{0}\right) \quad \Longrightarrow \quad\left|H_{z}(x, z)\right|^{\sigma} \leq C_{0} \hat{H}(x, z)|z|^{\sigma}
$$

(S3) $H_{z}(x, z)=V_{\infty}(x) z+\bar{H}_{z}(x, z)$, where $V_{\infty} \in C\left(\mathbb{R}^{N}\right)$ is 1-periodic in each of $x_{1}, x_{2}, \ldots, x_{N}$ with $\inf V_{\infty}>0$, and there exists $z_{0} \in E^{+} \backslash\{0\}$ such that

$$
\tau^{2}\left\|z_{0}\right\|^{2}-\|w\|^{2}-\int_{\mathbb{R}^{N}} V_{\infty}(x)\left(\tau z_{0}+w\right)^{2} \mathrm{~d} x<0, \quad \forall \tau>0, w \in E^{-}
$$

$H_{z}(x, z) \cdot \bar{H}_{z}(x, z) \leq 0$ and $H_{z}(x, z) \cdot \bar{H}_{z}(x, z)<0$ for $0<|z| \leq \alpha_{0}$ for some $\alpha_{0}>0$, $\left|\bar{H}_{z}(x, z)\right|=o(|z|)$ as $|z| \rightarrow \infty$ uniformly in $x \in \mathbb{R}^{N}$;

(S3') $H_{z}(x, z)=V_{\infty}(x) z+\bar{H}_{z}(x, z)$, where $V_{\infty} \in C\left(\mathbb{R}^{N}\right)$ is 1-periodic in each of $x_{1}, x_{2}, \ldots, x_{N}$ with $\inf V_{\infty}>\Lambda,\left|\bar{H}_{z}(x, z)\right|=o(|z|)$ as $|z| \rightarrow \infty$ uniformly in $x \in \mathbb{R}^{N}$, and $H_{z}(x, z)$. $\bar{H}_{z}(x, z)<0$ for $x \in \mathbb{R}^{N}$ and $z \neq(0,0)$; 
(S4) For all $\theta \geq 0, x \in \mathbb{R}^{N}, z, \zeta \in E$, there holds

$$
\frac{1-\theta^{2}}{2} H_{z}(x, z) \cdot z-\theta H_{z}(x, z) \cdot \zeta+H(x, \theta z+\zeta)-H(x, z) \geq 0
$$

$\left(\mathrm{S} 4^{\prime}\right) \hat{H}(x, z) \geq 0$, and for any $x \in \mathbb{R}^{N}, z, \zeta \in \mathbb{R}^{2}$, there holds

$$
2 H_{z}(x, z) \cdot z[H(x, z)-H(x, \zeta)] \leq\left(H_{z}(x, z) \cdot z\right)^{2}-\left(H_{z}(x, z) \cdot \zeta\right)^{2}
$$

whenever $H_{z}(x, z) \cdot \zeta=H_{z}(x, \zeta) \cdot z \neq 0$.

Under (V), (S0), and (S1), it follows from Lemma 2.2 that, for any $\varepsilon>0$, there exist $C_{\varepsilon}>0$ and $p \in\left(2,2^{*}\right)$ such that

$$
\left|H_{z}(x, z)\right| \leq \varepsilon|z|+C_{\varepsilon}|z|^{p-1}, \quad \forall(x, z) \in \mathbb{R}^{N} \times \mathbb{R}^{2}
$$

Clearly, (S0) holds under (S1) and (S3), as well as under (S1), (S2), and (SQ). For any $z=$ $(u, v) \in E$, it follows from $(2.6)$ and $(2.15)$ that $\eta=(\varphi, \psi)=\left(\frac{u+v}{\sqrt{2}}, \frac{u-v}{\sqrt{2}}\right)$ and

$$
\begin{aligned}
\int_{\mathbb{R}^{N}} & (\nabla \varphi \nabla \psi+V(x) \varphi \psi) \mathrm{d} x \\
= & \frac{1}{2} \int_{\mathbb{R}^{N}}\left(|\nabla u|^{2}+V(x)|u|^{2}-|\nabla v|^{2}-V(x)|v|^{2}\right) \mathrm{d} x \\
= & \frac{1}{2}\left(\left\|u^{+}\right\|_{H}^{2}-\left\|u^{-}\right\|_{H}^{2}-\left\|v^{+}\right\|_{H}^{2}+\left\|v^{-}\right\|_{H}^{2}\right) \\
= & \frac{1}{2}\left(\left\|z^{+}\right\|^{2}-\left\|z^{-}\right\|^{2}\right) .
\end{aligned}
$$

Thus, we have an equivalent functional

$$
\Phi(z)=\Phi(u, v)=\frac{1}{2}\left(\left\|z^{+}\right\|^{2}-\left\|z^{-}\right\|^{2}\right)-\Psi(z)
$$

and

$$
\left\langle\Phi^{\prime}(z), \zeta\right\rangle=\left(z^{+}, \zeta^{+}\right)-\left(z^{-}, \zeta^{-}\right)-\left\langle\Psi^{\prime}(z), \zeta\right\rangle, \quad \forall z, \zeta \in E
$$

where

$$
\Psi(z)=\int_{\mathbb{R}^{N}} H(x, z) \mathrm{d} x, \quad\left\langle\Psi^{\prime}(z), \zeta\right\rangle=\int_{\mathbb{R}^{N}} H_{z}(x, z) \cdot \zeta \mathrm{d} x .
$$

It is obvious that $z=(u, v) \in E$ is a critical point of $\Phi$ if and only if $\left(\frac{u+v}{\sqrt{2}}, \frac{u-v}{\sqrt{2}}\right)$ is a critical point of $I$. In what follows, we shall seek for the critical points of $\Phi$ under the assumptions on $H$. Obviously, the sets $\mathcal{M}$ and $\mathcal{N}^{-}$defined by (1.2) and (1.3) can be rewritten as follows:

$$
\mathcal{M}:=\left\{z \in E \backslash\{(0,0)\}: \Phi^{\prime}(z)=0\right\}
$$

and

$$
\mathcal{N}^{-}=\left\{z \in E \backslash E^{-}:\left\langle\Phi^{\prime}(z), z\right\rangle=\left\langle\Phi^{\prime}(z), \zeta\right\rangle=0, \forall \zeta \in E^{-}\right\}
$$


By (V), the functional $\Phi$ is strongly indefinite, the type of which has been largely investigated by authors via critical point theory, see $[8,12,13,18,33,37]$ and the references therein.

Before proceeding to the proof of the main results, we need some preliminaries.

Lemma 2.4 Suppose that (V), (S0), and (S1) are satisfied. If inf $V_{\infty}>0$, then

$$
\begin{aligned}
\tau\left|\Phi^{\prime}(z), \tau z+2 \zeta\right\rangle \geq & \tau^{2}\left\|z^{+}\right\|^{2}-\left\|\tau z^{-}+\zeta\right\|^{2}+\|\zeta\|^{2}-\int_{\mathbb{R}^{N}} V_{\infty}(x)|\tau z+\zeta|^{2} \mathrm{~d} x \\
& +\tau^{2} \int_{\mathbb{R}^{N}} \frac{z \cdot H_{z}(x, z) V_{\infty}(x)-\left|H_{z}(x, z)\right|^{2}}{V_{\infty}(x)} \mathrm{d} x \\
& \forall z \in E, \tau \in \mathbb{R}, \zeta \in E^{-}
\end{aligned}
$$

Proof In view of (2.19) and inf $V_{\infty}>0$, we have

$$
\begin{aligned}
& \tau\left\langle\Phi^{\prime}(z), \tau z+2 \zeta\right\rangle \\
& =\tau^{2}\left\|z^{+}\right\|^{2}-\tau^{2}\left\|z^{-}\right\|^{2}-2 \tau\left(z^{-}, \zeta\right)-\tau \int_{\mathbb{R}^{N}} H_{z}(x, z) \cdot(\tau z+2 \zeta) \mathrm{d} x \\
& =\tau^{2}\left\|z^{+}\right\|^{2}-\left\|\tau z^{-}+\zeta\right\|^{2}+\|\zeta\|^{2}-\int_{\mathbb{R}^{N}} V_{\infty}(x)|\tau z+\zeta|^{2} \mathrm{~d} x \\
& +\int_{\mathbb{R}^{N}}\left[V_{\infty}(x)|\tau z+\zeta|^{2}-\tau H_{z}(x, z) \cdot(\tau z+2 \zeta)\right] \mathrm{d} x \\
& =\tau^{2}\left\|z^{+}\right\|^{2}-\left\|\tau z^{-}+\zeta\right\|^{2}+\|\zeta\|^{2}-\int_{\mathbb{R}^{N}} V_{\infty}(x)|\tau z+\zeta|^{2} \mathrm{~d} x \\
& +\int_{\mathbb{R}^{N}}\left\{V_{\infty}(x)|\zeta|^{2}+2 \tau\left[V_{\infty}(x) z-H_{z}(x, z)\right] \cdot \zeta\right. \\
& \left.+\left[V_{\infty}(x)|z|^{2}-z \cdot H_{z}(x, z)\right] \tau^{2}\right\} \mathrm{d} x \\
& \geq \tau^{2}\left\|z^{+}\right\|^{2}-\left\|\tau z^{-}+\zeta\right\|^{2}+\|\zeta\|^{2}-\int_{\mathbb{R}^{N}} V_{\infty}(x)|\tau z+\zeta|^{2} \mathrm{~d} x \\
& +\int_{\mathbb{R}^{N}} \frac{\left|V_{\infty}(x) \zeta+\tau\left[V_{\infty}(x) z-H_{z}(x, z)\right]\right|^{2}}{V_{\infty}(x)} \mathrm{d} x \\
& +\tau^{2} \int_{\mathbb{R}^{N}} \frac{z \cdot H_{z}(x, z) V_{\infty}(x)-\left|H_{z}(x, z)\right|^{2}}{V_{\infty}(x)} \mathrm{d} x \\
& \geq \tau^{2}\left\|z^{+}\right\|^{2}-\left\|\tau z^{-}+\zeta\right\|^{2}+\|\zeta\|^{2}-\int_{\mathbb{R}^{N}} V_{\infty}(x)|\tau z+\zeta|^{2} \mathrm{~d} x \\
& +\tau^{2} \int_{\mathbb{R}^{N}} \frac{z \cdot H_{z}(x, z) V_{\infty}(x)-\left|H_{z}(x, z)\right|^{2}}{V_{\infty}(x)} \mathrm{d} x, \quad \forall z \in E, \tau \in \mathbb{R}, \zeta \in E^{-},
\end{aligned}
$$

which shows that (2.23) holds.

Corollary 2.5 Suppose that (V), (S0), and (S1) are satisfied, and that inf $V_{\infty}>0$. Then

$$
\begin{aligned}
& \left\|z^{+}\right\|^{2}-\left\|z^{-}+\zeta\right\|^{2}-\int_{\mathbb{R}^{N}} V_{\infty}(x)|z+\zeta|^{2} \mathrm{~d} x \\
& \quad \leq-\|\zeta\|^{2}-\int_{\mathbb{R}^{N}} \frac{z \cdot H_{z}(x, z) V_{\infty}(x)-\left|H_{z}(x, z)\right|^{2}}{V_{\infty}(x)} \mathrm{d} x, \quad \forall z \in \mathcal{N}^{-}, \zeta \in E^{-} .
\end{aligned}
$$


Employing a standard argument (see [37] and [40]), one can easily check the following lemma.

Lemma 2.6 Let (V), (S0), and (S1) be satisfied. Then $\Psi$ is nonnegative and weakly sequentially lower semi-continuous, and $\Psi^{\prime}$ is weakly sequentially continuous.

\section{Proof of main results}

Lemma 3.1 Let (V), (S0), (S1), and (S4) be satisfied. Then, for any $z \in E$,

$$
\Phi(z) \geq \Phi(\tau z+\zeta)+\frac{1}{2}\|\zeta\|^{2}+\frac{1-\tau^{2}}{2}\left\langle\Phi^{\prime}(z), z\right\rangle-\tau\left\langle\Phi^{\prime}(z), \zeta\right\rangle, \quad \forall \zeta \in E^{-}, \tau \geq 0 .
$$

Proof The proof is the same as the one of [35, Lemma 3.3], here we omit the details.

From Lemma 3.1, we have the following two corollaries.

Corollary 3.2 Let (V), (S0), (S1), and (S4) be satisfied. Then, for any $z \in \mathcal{N}^{-}$,

$$
\Phi(z) \geq \Phi(\tau z+\zeta)+\frac{1}{2}\|\zeta\|^{2}, \quad \forall \zeta \in E^{-}, \tau \geq 0 .
$$

Corollary 3.3 Let (V), (S0), (S1), and (S4) be satisfied. Then, for any $z \in E$,

$$
\Phi(z) \geq \Phi\left(\tau z^{+}\right)+\frac{\tau^{2}\left\|z^{-}\right\|^{2}}{2}+\frac{1-\tau^{2}}{2}\left\langle\Phi^{\prime}(z), z\right\rangle+\tau^{2}\left\langle\Phi^{\prime}(z), z^{-}\right\rangle, \quad \forall \tau \geq 0 .
$$

Applying Corollary 3.2, we can prove the following lemma in the same way as [33, Lemma 2.6].

Lemma 3.4 Let (V), (S0), (S1), and (S4) be satisfied. Then

(i) there exists $\rho>0$ such that $m:=\inf _{\mathcal{N}^{-}} \Phi \geq \kappa:=\inf \left\{\Phi(z): z \in E^{+},\|z\|=\rho\right\}>0$;

(ii) $\left\|z^{+}\right\| \geq \max \left\{\left\|z^{-}\right\|, \sqrt{2 m}\right\}$ for all $z \in \mathcal{N}^{-}$.

Define a set $E_{0}^{+}$as follows:

$$
E_{0}^{+}=\left\{z \in E^{+} \backslash\{0\}:\|z\|^{2}-\|\zeta\|^{2}-\int_{\mathbb{R}^{N}} V_{\infty}(x)|z+\zeta|^{2} \mathrm{~d} x<0, \forall \zeta \in E^{-}\right\} .
$$

Obviously, (S3) shows that the set $E_{0}^{+}$is not empty.

Lemma 3.5 Let (V), (S1), and (S3) be satisfied. Then, for any $e \in E_{0}^{+}$, there is a constant $r_{1}>\rho$ such that $\sup \Phi(\partial Q) \leq 0$ for $r \geq r_{1}$, where

$$
Q=\left\{\zeta+s e: \zeta \in E^{-}, s \geq 0,\|\zeta+s e\| \leq r\right\}
$$

Proof It is sufficient to show $\Phi(z) \leq 0$ for $z \in E^{-} \oplus \mathbb{R} e$ and $\|z\| \geq R$ for large $R$. Arguing indirectly, assume that, for some sequence $\left\{z_{n}\right\} \subset E^{-} \oplus \mathbb{R}^{+} e$ with $\left\|z_{n}\right\| \rightarrow \infty, \Phi\left(z_{n}\right) \geq 0$ for all $n \in \mathbb{N}$. Set $w_{n}=\frac{z_{n}}{\left\|z_{n}\right\|}=w_{n}^{-}+s_{n} e$, then $\left\|w_{n}\right\|=1$. Passing to a subsequence, we may assume that $w_{n} \rightarrow w$ in $E$. By Lemma 2.1 and (2.18), then $w_{n} \rightarrow w$ a.e. on $\mathbb{R}^{N}, s_{n} \rightarrow s$ and

$$
0 \leq \frac{\Phi\left(z_{n}\right)}{\left\|z_{n}\right\|^{2}}=\frac{1}{2} s_{n}^{2}\|e\|^{2}-\frac{1}{2}\left\|w_{n}^{-}\right\|^{2}-\int_{\mathbb{R}^{N}} \frac{H\left(x, z_{n}\right)}{\left|z_{n}\right|^{2}}\left|w_{n}\right|^{2} \mathrm{~d} x .
$$


If $s=0$, it follows from (S1) and (3.6) that

$$
0 \leq \frac{1}{2}\left\|w_{n}^{-}\right\|^{2}+\int_{\mathbb{R}^{N}} \frac{H\left(x, z_{n}\right)}{\left|z_{n}\right|^{2}}\left|w_{n}\right|^{2} \mathrm{~d} x \leq \frac{s_{n}^{2}}{2}\|e\|^{2} \rightarrow 0,
$$

which yields $\left\|w_{n}^{-}\right\| \rightarrow 0$, and so $1=\left\|w_{n}\right\| \rightarrow 0$, a contradiction.

If $s \neq 0$, then $w \neq 0$. Since $e \in E_{0}^{+}$, there exists a bounded domain $\Omega \subset \mathbb{R}^{N}$ such that

$$
s^{2}\|e\|^{2}-\left\|w^{-}\right\|^{2}-\int_{\Omega} V_{\infty}(x)\left|s e+w^{-}\right|^{2} \mathrm{~d} x<0 .
$$

By (S3), $H(x, z)=\frac{1}{2} V_{\infty}(x)|z|^{2}+\bar{H}(x, z)$. It follows from (3.6) that

$$
\begin{aligned}
0 & \leq \frac{s_{n}^{2}}{2}\|e\|^{2}-\frac{1}{2}\left\|w_{n}^{-}\right\|^{2}-\int_{\Omega} \frac{H\left(x, z_{n}\right)}{\left\|z_{n}\right\|^{2}} \mathrm{~d} x \\
& =\frac{s_{n}^{2}}{2}\|e\|^{2}-\frac{1}{2}\left\|w_{n}^{-}\right\|^{2}-\frac{1}{2} \int_{\Omega} V_{\infty}(x)\left|w_{n}\right|^{2} \mathrm{~d} x-\int_{\Omega} \frac{\bar{H}\left(x, z_{n}\right)}{\left\|z_{n}\right\|^{2}} \mathrm{~d} x .
\end{aligned}
$$

Clearly, $|\bar{H}(x, z)| \leq C_{1}|z|^{2}$ for some $C_{1}>0$ and $|\bar{H}(x, z)| /|z|^{2} \rightarrow 0$ as $|z| \rightarrow \infty$. Since $w_{n} \rightarrow w$ in $E$, then $w_{n} \rightarrow w$ in $L^{2}(\Omega)$, and it is easy to see from the Lebesgue dominated convergence theorem that

$$
\int_{\Omega} \frac{H\left(x, z_{n}\right)}{\left\|z_{n}\right\|^{2}} \mathrm{~d} x=\int_{\Omega} \frac{F\left(x, z_{n}\right)}{\left|z_{n}\right|^{2}}\left|w_{n}\right|^{2} \mathrm{~d} x=o(1) .
$$

Hence

$$
0 \leq s^{2}\|e\|^{2}-\left\|w^{-}\right\|^{2}-\int_{\Omega} V_{\infty}(x)\left|s e+w^{-}\right|^{2} \mathrm{~d} x
$$

a contradiction to (3.7).

Lemma 3.6 Let (V), (S1), (S2), and (SQ) be satisfied. Then, for any $e \in E^{+}$with $\|e\|=1$, there is a constant $r_{1}>\rho$ such that $\sup \Phi(\partial Q) \leq 0$ for $r \geq r_{1}$, where

$$
Q=\left\{\zeta+s e: \zeta \in E^{-}, s \geq 0,\|\zeta+s e\| \leq r\right\}
$$

Proof The argument is similar to the proof of Lemma 3.5, we only need to modify the last part of it. Indeed, for the case $s \neq 0$, it follows from (S1), (SQ), (3.6), and Fatou's lemma that

$$
\begin{aligned}
0 & \leq \limsup _{n \rightarrow \infty}\left[\frac{s_{n}^{2}}{2}-\frac{1}{2}\left\|w_{n}^{-}\right\|^{2}-\int_{\mathbb{R}^{N}} \frac{H\left(x, z_{n}\right)}{\left|z_{n}\right|^{2}}\left|w_{n}\right|^{2} \mathrm{~d} x\right] \\
& \leq \lim _{n \rightarrow \infty} \frac{s_{n}^{2}}{2}-\liminf _{n \rightarrow \infty} \int_{\mathbb{R}^{N}} \frac{H\left(x, z_{n}\right)}{\left|z_{n}\right|^{2}}\left|w_{n}\right|^{2} \mathrm{~d} x \\
& \leq \frac{s^{2}}{2}-\int_{\mathbb{R}^{N}} \liminf _{n \rightarrow \infty} \frac{H\left(x, z_{n}\right)}{\left|z_{n}\right|^{2}}\left|w_{n}\right|^{2} \mathrm{~d} x=-\infty,
\end{aligned}
$$

a contradiction. 
By virtue of Lemmas 3.4 and 3.5, one can prove the following lemma by using the same argument as in the proof of [33, Lemma 2.6].

Lemma 3.7 Let (V), (S1), (S3), and (S4) be satisfied. Then, for any $z \in E_{0}^{+}, \mathcal{N}^{-} \cap\left(E^{-} \oplus\right.$ $\left.\mathbb{R}^{+} z\right) \neq \emptyset$, i.e., there exist $\tau(z)>0$ and $w(z) \in E^{-}$such that $\tau(z) z+w(z) \in \mathcal{N}^{-}$.

Applying Lemmas 2.3, 2.6, 3.4(i), 3.5, and 3.6, one gets directly the following.

Lemma 3.8 Under (V) and (S1), either (S2), (SQ) are satisfied or (S3) holds. Then there exist a constant $c \in[\kappa, \sup \Phi(Q)]$ and a sequence $\left\{z_{n}\right\} \subset E$ such that

$$
\Phi\left(z_{n}\right) \rightarrow c, \quad\left\|\Phi^{\prime}\left(z_{n}\right)\right\|\left(1+\left\|z_{n}\right\|\right) \rightarrow 0,
$$

where $Q$ is defined by (3.8).

The following lemma shows that a minimizing Cerami sequence for the energy functional can be found outside the Nehari-Pankov manifold, from which one can easily demonstrate a ground state solution of Nehari-Pankov type for problem (1.1).

Lemma 3.9 Let (V), (S1), (S3), and (S4) be satisfied. Then there exist a constant $c_{*} \in[\kappa, m]$ and $a$ sequence $\left\{z_{n}\right\} \subset E$ satisfying

$$
\Phi\left(z_{n}\right) \rightarrow c_{*}, \quad\left\|\Phi^{\prime}\left(z_{n}\right)\right\|\left(1+\left\|z_{n}\right\|\right) \rightarrow 0 .
$$

Proof The proof is essentially contained in [34], we omit it here.

Lemma 3.10 Let (V), (S1), and (S3) be satisfied. Then for any sequence $\left\{z_{n}\right\} \subset$ E satisfying (3.10) is bounded in $E$.

Proof To prove the boundedness of $\left\{z_{n}\right\}$, arguing by contradiction, suppose that $\left\|z_{n}\right\| \rightarrow$ $\infty$. Let $w_{n}=z_{n} /\left\|z_{n}\right\|$, then $\left\|w_{n}\right\|=1$. By Lemma 2.1, there exists a constant $C_{2}>0$ such that $\left\|w_{n}^{+}\right\|_{2} \leq C_{2}$. If

$$
\delta:=\limsup _{n \rightarrow \infty} \sup _{y \in \mathbb{R}^{N}} \int_{B_{1}(y)}\left|w_{n}^{+}\right|^{2} \mathrm{~d} x=0,
$$

then by Lions's concentration compactness principle [24] or [37, Lemma 1.21] (usually this lemma is stated for $\left\{z_{n}\right\} \subset H^{1}\left(\mathbb{R}^{N}\right)$; however, a simple modification of the argument in [24] shows that the conclusion remains valid for $E), w_{n}^{+} \rightarrow 0$ in $L^{s}\left(\mathbb{R}^{N}, \mathbb{R}^{2}\right)$ for $2<s<2^{*}$. Fix $R>[2(1+c)]^{1 / 2}$. By virtue of $(2.16)$, for $\varepsilon=1 / 4\left(R C_{2}\right)^{2}>0$, we have

$$
\limsup _{n \rightarrow \infty} \int_{\mathbb{R}^{N}} H\left(x, R w_{n}^{+}\right) \mathrm{d} x \leq \varepsilon\left(R C_{2}\right)^{2}+R^{p} C_{\varepsilon} \lim _{n \rightarrow \infty}\left\|w_{n}^{+}\right\|_{p}^{p}=\frac{1}{4} .
$$

Let $\tau_{n}=R /\left\|z_{n}\right\|$. Hence, by virtue of (3.10), (3.11), and Corollary 3.3, one can get that

$$
\begin{aligned}
c+o(1)= & \Phi\left(z_{n}\right) \geq \frac{\tau_{n}^{2}}{2}\left(\left\|z_{n}^{+}\right\|^{2}+\left\|z_{n}^{-}\right\|^{2}\right)-\int_{\mathbb{R}^{N}} H\left(x, \tau_{n} z_{n}^{+}\right) \mathrm{d} x+\frac{1-\tau_{n}^{2}}{2}\left\langle\Phi^{\prime}\left(z_{n}\right), z_{n}\right\rangle \\
& +\tau_{n}^{2}\left\langle\Phi^{\prime}\left(z_{n}\right), z_{n}^{-}\right\rangle
\end{aligned}
$$




$$
\begin{aligned}
= & \frac{R^{2}}{2}\left(\left\|w_{n}^{+}\right\|^{2}+\left\|w_{n}^{-}\right\|^{2}\right)-\int_{\mathbb{R}^{N}} H\left(x, R w_{n}^{+}\right) \mathrm{d} x+\left(\frac{1}{2}-\frac{R^{2}}{2\left\|z_{n}\right\|^{2}}\right)\left\langle\Phi^{\prime}\left(z_{n}\right), z_{n}\right\rangle \\
& +\frac{R^{2}}{\left\|z_{n}\right\|^{2}}\left\langle\Phi^{\prime}\left(z_{n}\right), z_{n}^{-}\right\rangle \\
= & \frac{R^{2}}{2}-\int_{\mathbb{R}^{N}} H\left(x, R w_{n}^{+}\right) \mathrm{d} x+o(1) \geq \frac{R^{2}}{2}-\frac{1}{4}+o(1)>c+\frac{3}{4}+o(1) .
\end{aligned}
$$

This contradiction shows that $\delta>0$.

Going if necessary to a subsequence, we may assume the existence of $k_{n} \in \mathbb{Z}^{N}$ such that $\int_{B_{1+\sqrt{N}}\left(k_{n}\right)}\left|w_{n}^{+}\right|^{2} \mathrm{~d} x>\frac{\delta}{2}$. Let $\tilde{w}_{n}(x)=w_{n}\left(x+k_{n}\right)$. Since $V(x)$ is 1 -periodic in $x$, then $\left\|\tilde{w}_{n}\right\|=$ $\left\|w_{n}\right\|=1$, and

$$
\int_{B_{1+\sqrt{N}}(0)}\left|\tilde{w}_{n}^{+}\right|^{2} \mathrm{~d} x>\frac{\delta}{2}
$$

Passing to a subsequence, we have $\tilde{w}_{n} \rightarrow \tilde{w}$ in $E, \tilde{w}_{n} \rightarrow \tilde{w}$ in $L_{\operatorname{loc}}^{s}\left(\mathbb{R}^{N}, \mathbb{R}^{2}\right), 2 \leq s<2^{*}, \tilde{w}_{n} \rightarrow$ $\tilde{w}$ a.e. on $\mathbb{R}^{N}$. It follows from (3.12) that $\tilde{w}^{+} \neq 0$, and so $\tilde{w} \neq 0$.

Now we define $\tilde{z}_{n}(x)=z_{n}\left(x+k_{n}\right)$, then $\tilde{z}_{n} /\left\|z_{n}\right\|=\tilde{w}_{n} \rightarrow \tilde{w}$ a.e. on $\mathbb{R}^{N}, \tilde{w} \neq 0$. For $x \in$ $\Omega:=\left\{y \in \mathbb{R}^{N}: \tilde{w}(y) \neq 0\right\}$, we have $\lim _{n \rightarrow \infty}\left|\tilde{z}_{n}(x)\right|=\infty$. For any $\varsigma \in C_{0}^{\infty}\left(\mathbb{R}^{N}, \mathbb{R}^{2}\right)$, setting $\varsigma_{n}(x)=\varsigma\left(x-k_{n}\right)$, then

$$
\begin{aligned}
\left\langle\Phi^{\prime}\left(z_{n}\right), \varsigma_{n}\right\rangle & =\left(z_{n}^{+}-z_{n}^{-}, \varsigma_{n}\right)-\left(V_{\infty} z_{n}, \varsigma_{n}\right)_{2}-\int_{\mathbb{R}^{N}} \bar{H}_{z}\left(x, z_{n}\right) \cdot \varsigma_{n} \mathrm{~d} x \\
& =\left\|z_{n}\right\|\left[\left(w_{n}^{+}-w_{n}^{-}, \varsigma_{n}\right)-\left(V_{\infty} w_{n}, \varsigma_{n}\right)_{2}-\int_{\mathbb{R}^{N}} \frac{\bar{H}_{z}\left(x, z_{n}\right)}{\left|z_{n}\right|} \cdot \varsigma_{n}\left|w_{n}\right| \mathrm{d} x\right] \\
& =\left\|z_{n}\right\|\left[\left(\tilde{w}_{n}^{+}-\tilde{w}_{n}^{-}, \varsigma\right)-\left(V_{\infty} \tilde{w}_{n}, \varsigma\right)_{2}-\int_{\mathbb{R}^{N}} \frac{\bar{H}_{z}\left(x, \tilde{z}_{n}\right)}{\left|\tilde{z}_{n}\right|} \cdot \varsigma\left|\tilde{w}_{n}\right| \mathrm{d} x\right],
\end{aligned}
$$

which, together with (3.10), yields that

$$
\left(\tilde{w}_{n}^{+}-\tilde{w}_{n}^{-}, \varsigma\right)-\left(V_{\infty} \tilde{w}_{n}, \varsigma\right)_{2}-\int_{\mathbb{R}^{N}} \frac{\bar{H}_{z}\left(x, \tilde{z}_{n}\right)}{\left|\tilde{z}_{n}\right|} \cdot \varsigma\left|\tilde{w}_{n}\right| \mathrm{d} x=o(1) .
$$

Note that, for some constant $C>0$, there holds

$$
\begin{aligned}
\left|\int_{\mathbb{R}^{N}} \frac{\bar{H}_{z}\left(x, \tilde{z}_{n}\right)}{\left|\tilde{z}_{n}\right|} \cdot \varsigma\right| \tilde{w}_{n}|\mathrm{~d} x| & \leq \int_{\mathbb{R}^{N}} \frac{\bar{H}_{z}\left(x, \tilde{z}_{n}\right)}{\left|\tilde{z}_{n}\right|}|| \tilde{w}_{n}-\tilde{w}|| \varsigma\left|\mathrm{d} x+\int_{\mathbb{R}^{N}}\right| \frac{\bar{H}_{z}\left(x, \tilde{z}_{n}\right)}{\tilde{z}_{n}}|| \tilde{w}|\varsigma| \mathrm{d} x \\
& \leq C \int_{\text {supp } \varsigma}\left|\tilde{w}_{n}-\tilde{w}\right||\varsigma| \mathrm{d} x+\int_{\Omega}\left|\frac{\bar{H}_{z}\left(x, \tilde{z}_{n}\right)}{\tilde{z}_{n}}\right||\tilde{w}||\varsigma| \mathrm{d} x=o(1) .
\end{aligned}
$$

Hence,

$$
\left(\tilde{w}^{+}-\tilde{w}^{-}, \varsigma\right)-\left(V_{\infty} \tilde{w}, \varsigma\right)_{2}=0, \quad \forall \varsigma=(\varphi, \psi) \in C_{0}^{\infty}\left(\mathbb{R}^{N}, \mathbb{R}^{2}\right)
$$

Let $\tilde{w}=(u, v)$, then $\tilde{w}^{+}-\tilde{w}^{-}=\left(u^{+}-u^{-}, v^{-}-v^{+}\right)$. Thus from (3.14) we have

$$
\left(u^{+}-u^{-}, \varphi\right)_{H}+\left(v^{-}-v^{+}, \psi\right)_{H}-\left(V_{\infty} u, \varphi\right)_{L^{2}}-\left(V_{\infty} v, \psi\right)_{L^{2}}=0,
$$


which implies that

$$
\left\{\begin{array}{l}
-\Delta u+V(x) u=V_{\infty} u \\
\Delta v+V(x) v=V_{\infty} v .
\end{array}\right.
$$

Hence $u$ is an eigenfunction of operator $\mathcal{B}_{1}:=-\Delta+\left(V-V_{\infty}\right)$, and $v$ is an eigenfunction of operator $\mathcal{B}_{2}:=-\triangle+\left(V_{\infty}-V\right)$, which contradicts the fact that $\mathcal{B}_{i}$ has only continuous spectrum for $i=1,2$ since $V-V_{\infty}$ is 1 -periodic. This contradiction shows that $\left\{z_{n}\right\}$ is bounded.

Lemma 3.11 Let (V), (S1), (S2), and (SQ) be satisfied. Then for any sequence $\left\{z_{n}\right\} \subset E$ satisfying

$$
\Phi\left(z_{n}\right) \rightarrow c \geq 0, \quad\left\langle\Phi^{\prime}\left(z_{n}\right), z_{n}^{ \pm}\right\rangle \rightarrow 0
$$

is bounded in $E$.

Proof By virtue of (S4) and (3.15), there exists a constant $C_{3}>0$ such that

$$
C_{3} \geq \Phi\left(z_{n}\right)-\frac{1}{2}\left\langle\Phi^{\prime}\left(z_{n}\right), z_{n}\right\rangle=\int_{\mathbb{R}^{N}} \hat{H}\left(x, z_{n}\right) \mathrm{d} x \geq 0 .
$$

To prove the boundedness of $\left\{z_{n}\right\}$, arguing by contradiction, suppose that $\left\|z_{n}\right\| \rightarrow \infty$. Let $w_{n}=z_{n} /\left\|z_{n}\right\|$, then $\left\|w_{n}\right\|=1$. If $\delta:=\limsup _{n \rightarrow \infty} \sup _{y \in \mathbb{R}^{N}} \int_{B_{1}(y)}\left|w_{n}^{+}\right|^{2} \mathrm{~d} x=0$, then by Lions's concentration compactness principle, $w_{n}^{+} \rightarrow 0$ in $L^{s}\left(\mathbb{R}^{N}, \mathbb{R}^{2}\right)$ for $2<s<2^{*}$. Set $\sigma^{\prime}=\sigma /(\sigma-$ 1) and

$$
\Omega_{n}:=\left\{x \in \mathbb{R}^{N}: \frac{\left|H_{z}(x, z)\right|}{|z|} \leq \frac{1}{2}\left(\Lambda_{0}-\delta_{0}\right)\right\} .
$$

Then $2 \sigma^{\prime} \in\left(2,2^{*}\right)$ since $\sigma>\max \{1, N / 2\}$. Note that $\Lambda_{0}\|z\|_{2}^{2} \leq\|z\|^{2}$ for any $z \in E$, then

$$
\int_{\Omega_{n}} \frac{\left|H_{z}\left(x, z_{n}\right)\right|}{\left|z_{n}\right|}\left|w_{n}\right|\left|w_{n}^{+}\right| \mathrm{d} x \leq \frac{1}{2}\left(\Lambda_{0}-\delta_{0}\right)\left\|w_{n}^{+}\right\|_{2}\left\|w_{n}\right\|_{2} \leq \frac{1}{2}-\frac{\delta_{0}}{2 \Lambda_{0}} .
$$

On the other hand, by virtue of (S4), (3.16), Lemma 2.1, and the Hölder inequality, there exists a constant $C_{4}>0$ such that

$$
\begin{aligned}
\int_{\mathbb{R}^{N} \backslash \Omega_{n}} \frac{\left|H_{z}\left(x, z_{n}\right)\right|}{\left|z_{n}\right|}\left|w_{n}\right|\left|w_{n}^{+}\right| \mathrm{d} x & \leq\left[\int_{\mathbb{R}^{N} \backslash \Omega_{n}}\left(\frac{\left|H_{z}\left(x, z_{n}\right)\right|}{\left|z_{n}\right|}\right)^{\sigma}\right]^{1 / \sigma}\left\|w_{n}^{+}\right\|_{2 \sigma^{\prime}}\left\|w_{n}\right\|_{2 \sigma^{\prime}} \\
& \leq C_{4}\left(\int_{\mathbb{R}^{N} \backslash \Omega_{n}} C_{0} \hat{H}\left(x, z_{n}\right) \mathrm{d} x\right)^{1 / \sigma}\left\|w_{n}^{+}\right\|_{2 \sigma^{\prime}} \\
& \leq C_{4}\left(C_{0} C_{3}\right)^{1 / \sigma}\left\|w_{n}^{+}\right\|_{2 \sigma^{\prime}}=o(1) .
\end{aligned}
$$

In view of (S1), (2.18), and (3.15), one has

$$
2 c+o(1)=\left\|z_{n}^{+}\right\|^{2}-\left\|z_{n}^{-}\right\|^{2}-2 \int_{\mathbb{R}^{N}} H\left(x, z_{n}\right) \mathrm{d} x \leq\left\|z_{n}^{+}\right\|^{2}-\left\|z_{n}^{-}\right\|^{2} .
$$


Combining (3.18) and (3.19) and using (2.19), (3.15), and (3.20), we have

$$
\begin{aligned}
\frac{1}{2}+o(1) & \leq \frac{\left\|z_{n}^{+}\right\|^{2}-\left\langle\Phi^{\prime}\left(z_{n}\right), z_{n}^{+}\right\rangle}{\left\|z_{n}\right\|^{2}} \\
& =\frac{1}{\left\|z_{n}\right\|} \int_{\left|z_{n}^{+}\right| \neq 0} \frac{H_{z}\left(x, z_{n}\right) \cdot z_{n}^{+}}{\left|z_{n}^{+}\right|}\left|w_{n}^{+}\right| \mathrm{d} x \\
& \leq \int_{\left|z_{n}\right| \neq 0} \frac{\left|H_{z}\left(x, z_{n}\right)\right|}{\left|z_{n}\right|}\left|w_{n}\right|\left|w_{n}^{+}\right| \mathrm{d} x \\
& \leq \int_{\Omega_{n}} \frac{\left|H_{z}\left(x, z_{n}\right)\right|}{\left|z_{n}\right|}\left|w_{n}\right|\left|w_{n}^{+}\right| \mathrm{d} x+\int_{\mathbb{R}^{N} \backslash \Omega_{n}} \frac{\left|H_{z}\left(x, z_{n}\right)\right|}{\left|z_{n}\right|}\left|w_{n}\right|\left|w_{n}^{+}\right| \mathrm{d} x \\
& \leq \frac{1}{2}-\frac{\delta_{0}}{2 \Lambda_{0}}+o(1) .
\end{aligned}
$$

This contradiction shows that $\delta>0$. The rest of the argument is standard, so we omit it.

We first prove Theorem 1.4, then sketch the proof of Theorem 1.3.

Proof of Theorem 1.4 Applying Lemmas 3.9 and 3.10, there exists a bounded sequence $\left\{z_{n}\right\} \subset E$ satisfying (3.10). By Lemma 2.1, there is a constant $C_{5}>0$ such that $\left\|z_{n}\right\|_{2}^{2}+$ $\left\|z_{n}\right\|_{p}^{p} \leq C_{5}$. If

$$
\delta:=\limsup _{n \rightarrow \infty} \sup _{y \in \mathbb{R}^{N}} \int_{B(y, 1)}\left|z_{n}\right| \mathrm{d} x=0,
$$

then by Lions's concentration compactness principle, $z_{n} \rightarrow 0$ in $L^{s}\left(\mathbb{R}^{N}, \mathbb{R}^{2}\right)$ for $2<s<2^{*}$. From (S1), (2.16), (2.18), (2.19), and (3.10), one has

$$
\begin{aligned}
2 c_{*}+o(1) & =\left\|z_{n}^{+}\right\|^{2}-\left\|z_{n}^{-}\right\|^{2}-2 \int_{\mathbb{R}^{N}} H\left(x, z_{n}\right) \mathrm{d} x \\
& \leq\left\|z_{n}^{+}\right\|^{2}=\left\langle\Phi^{\prime}\left(z_{n}\right), z_{n}^{+}\right\rangle+\int_{\mathbb{R}^{N}} H_{z}\left(x, z_{n}\right) z_{n}^{+} \mathrm{d} x \\
& \leq \varepsilon\left\|z_{n}\right\|_{2}\left\|z_{n}^{+}\right\|_{2}+C_{\varepsilon}\left\|z_{n}\right\|_{p}^{p-1}\left\|z_{n}^{+}\right\|_{p}+o(1) \\
& \leq \varepsilon C_{5}+o(1) .
\end{aligned}
$$

This is a contradiction since $\varepsilon>0$ is arbitrary. Thus $\delta>0$.

Going if necessary to a subsequence, we may assume the existence of $k_{n} \in \mathbb{Z}^{N}$ such that

$$
\int_{B\left(k_{n}, 1+\sqrt{N}\right)}\left|z_{n}\right|^{2} \mathrm{~d} x>\frac{\delta}{2}
$$

Let us define $w_{n}(x)=z_{n}\left(x+k_{n}\right)$ so that

$$
\int_{B(0,1+\sqrt{N})}\left|w_{n}\right|^{2}>\frac{\delta}{2} \text {. }
$$

Since $V(x), H(x, z)$ are periodic in $x$, together with (3.10), we have $\left\|w_{n}\right\|=\left\|z_{n}\right\|$ and

$$
\Phi\left(w_{n}\right) \rightarrow c_{*} \in[\kappa, m], \quad\left\|\Phi^{\prime}\left(w_{n}\right)\right\|\left(1+\left\|w_{n}\right\|\right) \rightarrow 0 .
$$


Passing to a subsequence, we have $w_{n} \rightarrow w_{0}$ in $E, w_{n} \rightarrow w_{0}$ in $L_{\text {loc }}^{s}\left(\mathbb{R}^{N}, \mathbb{R}^{2}\right)$ for $2 \leq s<2^{*}$ and $w_{n} \rightarrow w_{0}$ a.e. on $\mathbb{R}^{N}$. Hence it follows from (3.22) and (3.23) that $\Phi^{\prime}\left(w_{0}\right)=0$ and $w_{0} \neq 0$. This shows that $w_{0} \in \mathcal{N}^{-}$, and so $\Phi\left(w_{0}\right) \geq m$. On the other hand, by (S1),(S4), (2.18), (2.19), (3.23), and Fatou's lemma, we have

$$
\begin{aligned}
m & \geq c_{*}=\lim _{n \rightarrow \infty}\left[\Phi\left(w_{n}\right)-\frac{1}{2}\left\langle\Phi^{\prime}\left(w_{n}\right), w_{n}\right\rangle\right] \\
& =\lim _{n \rightarrow \infty} \int_{\mathbb{R}^{N}} \hat{H}\left(x, w_{n}\right) \mathrm{d} x \geq \int_{\mathbb{R}^{N}} \liminf _{n \rightarrow \infty} \hat{H}\left(x, w_{n}\right) \mathrm{d} x \\
& =\int_{\mathbb{R}^{N}} \hat{H}\left(x, w_{0}\right) \mathrm{d} x=\Phi\left(w_{0}\right)-\frac{1}{2}\left\langle\Phi^{\prime}\left(w_{0}\right), w_{0}\right\rangle \\
& =\Phi\left(w_{0}\right) .
\end{aligned}
$$

This shows that $\Phi\left(w_{0}\right) \leq m$, and so $\Phi\left(w_{0}\right)=m=\inf _{\mathcal{N}^{-}} \Phi \geq \kappa>0$ by Lemma 3.4(i).

Proof of Theorem 1.3 It follows from Lemma 2.2 that (S1), (SQ), and (S2) yield (S0). Applying Lemmas 3.8 and 3.11, there exists a bounded sequence $\left\{z_{n}\right\} \subset E$ satisfying (3.9). Similar to the argument as in the proof of Theorem 1.3, we can show that $\Phi^{\prime}(\bar{z})=0$ for some $\bar{z} \in E \backslash\{(0,0)\}$, i.e., $\mathcal{M} \neq 0$. Let $\hat{c}:=\inf _{\mathcal{M}} \Phi$. By (S4), for any $z \in \mathcal{M}$, one has

$$
\Phi(z)=\Phi(z)-\frac{1}{2}\left\langle\Phi^{\prime}(z), z\right\rangle=\int_{\mathbb{R}^{N}} \hat{H}(x, z) \mathrm{d} x \geq 0
$$

therefore $\hat{c} \geq 0$. Let $\left\{z_{n}\right\} \subset \mathcal{M}$ such that $\Phi\left(z_{n}\right) \rightarrow \hat{c}$. Then $\left\langle\Phi^{\prime}\left(z_{n}\right), \zeta\right\rangle=0$ for any $\zeta \in E$. It follows from Lemma 3.11 that $\left\{z_{n}\right\}$ is bounded in $E$. The rest of the argument is the same as in the proof of Theorem 1.4 by using (S2) instead of (S4).

\section{Appendix}

Here, we prove that (G4) holds under (G1), $\left(\mathrm{G}^{\prime}\right)$, and $\left(\mathrm{G}_{2}\right)$. It is equivalent to showing that $\left(\mathrm{S}^{\prime}\right)$ together with $(\mathrm{S} 1),\left(\mathrm{S}^{\prime}\right)$ yields $(\mathrm{S} 4)$. For any $z, \zeta \in \mathbb{R}^{2}$, let

$$
h(t):=\frac{1-t^{2}}{2} H_{z}(x, z) \cdot z-t H_{z}(x, z) \cdot \zeta+H(x, t z+\zeta)-H(x, z), \quad \forall t \geq 0 .
$$

It is sufficient to show that $h(t) \geq 0$ for $t \geq 0$. If $z=0$, then $h(t)=H(x, \zeta) \geq 0$ by (S1). Assume $z \neq 0$. It follows from (S1), (S4') and $z \cdot \bar{H}_{z}(x, z)<0$ for $z \neq 0$ (deduced from (S3')) that

$$
h(0)=\frac{1}{2} H_{z}(x, z) \cdot z-H(x, z)+H(x, \zeta) \geq 0, \quad \text { and } \quad h(t) \geq 0 \quad \text { for any } t \text { large. }
$$

Assume that $h(t)$ reaches a minimum at some point $t_{0} \in[0, \infty)$. Clearly, $h(t) \geq 0$ for any $t \geq 0$ if $t_{0}=0$. Assume $t_{0}>0$. Then $h^{\prime}\left(t_{0}\right)=0$, i.e.,

$$
H_{z}\left(x, t_{0} z+\zeta\right) \cdot z-H_{z}(x, z) \cdot\left(t_{0} z+\zeta\right)=0
$$

If $H_{z}\left(x, t_{0} z+\zeta\right) \cdot z=0,(\mathrm{~S} 1)$ and $\left(\mathrm{S} 4^{\prime}\right)$ imply that

$$
h\left(t_{0}\right)=\frac{1+t_{0}^{2}}{2} H_{z}(x, z) \cdot z-H(x, z)+H\left(x, t_{0} z+\zeta\right) \geq 0
$$


If $H_{z}\left(x, t_{0} z+\zeta\right) \cdot z \neq 0,\left(\mathrm{~S}^{\prime}\right)$ yields that $H_{z}(x, z) \cdot z \neq 0$. Using (S1) and (S4') again, we have

$$
\begin{aligned}
h\left(t_{0}\right) \geq & \frac{1-t_{0}^{2}}{2} H_{z}(x, z) \cdot z-t_{0} H_{z}(x, z) \cdot \zeta-\left[2 H_{z}(x, z) \cdot z\right]^{-1} \\
& \times\left\{\left[H_{z}(x, z) \cdot z\right]^{2}-\left[H_{z}(x, z) \cdot\left(t_{0} z+\zeta\right)\right]^{2}\right\} \\
\geq & {\left[2 H_{z}(x, z) \cdot z\right]^{-1}\left[t_{0} H_{z}(x, z) \cdot z-H_{z}(x, z) \cdot\left(t_{0} z+\zeta\right)\right]^{2} } \\
\geq & 0 .
\end{aligned}
$$

Then $h(t) \geq 0$ for $t \geq 0$, and so (S4) holds.

\section{Acknowledgements}

The authors would like to express sincere thanks to the anonymous referees for their careful reading of the manuscript and valuable comments and suggestions.

\section{Funding}

This work was supported by the NSFC (Nos. 11801574, 11571370, 11601525), NSF of Hunan Province (Nos. 2017JJ2213, 2019JJ50788), Research Foundation of Education Bureau of Hunan Province, China (No. 18A452), and Central South University Innovation-Driven Project for Young Scholars (No. 2019CX022), Huaihua University Scientific Research Project (No. HHUY2019-3)

\section{Availability of data and materials}

Not applicable.

\section{Competing interests}

The authors declare that they have no competing interests.

\section{Authors' contributions}

The authors declare that this study was independently finished. All authors read and approved the final manuscript.

\section{Authors' information}

Not applicable.

\section{Author details}

${ }^{1}$ Department of Mathematic and Applied Mathematics, Huaihua University, Huaihua, P.R. China. ${ }^{2}$ School of Mathematics and Statistics, Central South University, Changsha, P.R. China.

\section{Publisher's Note}

Springer Nature remains neutral with regard to jurisdictional claims in published maps and institutional affiliations.

Received: 10 December 2018 Accepted: 24 September 2019 Published online: 03 October 2019

\section{References}

1. Alves, C.O., Carrião, P.C., Miyagaki, O.H.: On the existence of positive solutions of a perturbed Hamiltonian system in $\mathbb{R}^{N}$. J. Math. Anal. Appl. 276, 673-690 (2002)

2. Ávila, A.I., Yang, J.: On the existence and shape of least energy solutions for some elliptic systems. J. Differ. Equ. 191, $348-376(2003)$

3. Ávila, A.l., Yang, J.: Multiple solutions of nonlinear elliptic systems. Nonlinear Differ. Equ. Appl. 12, 459-479 (2005)

4. Bartsch, T., De Figueiredo, D.G.: Infinitely many solutions of nonlinear elliptic systems. In: Progress in Nonlinear Differential Equations and Their Applications, vol. 35, pp. 51-67. Birkhäuser, Basel (1999)

5. Bartsch, T., Ding, Y.H.: Deformation theorems on non-metrizable vector spaces and applications to critical point theory. Math. Nachr. 279, 1-22 (2006)

6. Bartsch, T., Mederski, J.: Ground and bound state solutions of semilinear time-harmonic Maxwell equations in a bounded domain. Arch. Ration. Mech. Anal. 215, 283-306 (2015)

7. Benci, V., Rabinowitz, P.H.: Critical point theorems for indefinite functionals. Invent. Math. 52, 241-273 (1979)

8. De Figueiredo, D.G., Ding, Y.H.: Strongly indefinite functionals and multiple solutions of elliptic systems. Trans. Am. Math. Soc. 355, 2973-2989 (2003)

9. De Figueiredo, D.G., Felmer, P.L.: On superquadratic elliptic systems. Trans. Am. Math. Soc. 343, 97-116 (1994)

10. De Figueiredo, D.G., Yang, J.: Decay, symmetry and existence of solutions of semilinear elliptic systems. Nonlinear Anal. 33, 211-234 (1998)

11. De Figueiredo, D.G., Marcos do Ó, J., Ruf, B.: An Orlicz-space approach to superlinear elliptic systems. J. Funct. Anal. 224, 471-496 (2005)

12. Ding, Y.H.: Varitional Methods for Strongly Indefinite Problems. World Scientific, Singapore (2008)

13. Ding, Y.H., Lee, C.: Multiple solutions of Schrödinger equations with indefinite linear part and super or asymptotically linear terms. J. Differ. Equ. 222, 137-163 (2006) 
14. Ding, Y.H., Lin, F.H.: Semiclassical states of Hamiltonian systems of Schrödinger equations with subcritical and critical nonlinearities. J. Partial Differ. Equ. 19, 232-255 (2006)

15. Edmunds, D.E., Evans, W.D.: Spectral Theory and Differential Operators. Clarendon, Oxford (1987)

16. Egorov, Y., Kondratiev, V.: On Spectral Theory of Elliptic Operators. Birkhäuser, Basel (1996)

17. Hulshof, J., Van der Vorst, R.: Differential systems with strongly variational structure. J. Funct. Anal. 114, 32-58 (1993)

18. Kryszewski, W., Szulkin, A.: An infinite dimensional Morse theorem with applications. Trans. Am. Math. Soc. 349, 3184-3234 (1997)

19. Kryszewski, W., Szulkin, A.: Generalized linking theorem with an application to semilinear Schrödinger equations. Adv. Differ. Equ. 3, 441-472 (1998)

20. Li, G., Szulkin, A.: An asymptotically periodic equation with indefinite linear part. Commun. Contemp. Math. 4, 763-776 (2002)

21. Li, G., Yang, J.: Asymptotically linear elliptic systems. Commun. Partial Differ. Equ. 29, 925-954 (2004)

22. Liao, F.F., Tang, X.H., Zhang, J., Qin, D.D.: Semi-classical solutions of perturbed elliptic system with general superlinear nonlinearity. Bound. Value Probl. 2014, 208 (2014)

23. Liao, F.F., Tang, X.H., Zhang, J.: Existence of solutions for periodic elliptic system with general superlinear nonlinearity. Z. Angew. Math. Phys. 66, 689-701 (2015)

24. Lions, P.L.: The concentration-compactness principle in the calculus of variations. The locally compact case, part 2. Ann. Inst. Henri Poincaré, Anal. Non Linéaire 1, 223-283 (1984)

25. Liu, Z.L., Wang, Z.-Q.: On the Ambrosetti-Rabinowitz superlinear condition. Adv. Nonlinear Stud. 4, 561-572 (2004)

26. Mao, A.M., Luan, S.X., Ding, Y.H.: Periodic solutions for a class of first order superquadratic Hamiltonian system. J. Math Anal. Appl. 30, 584-596 (2007)

27. Pankov, A.: Periodic nonlinear Schrödinger equation with application to photonic crystals. Milan J. Math. 73, 259-287 (2005)

28. Pistoia, A., Ramos, M.: Locating the peaks of the least energy solutions to an elliptic system with Neumann boundary conditions. J. Differ. Equ. 201, 160-176 (2004)

29. Qin, D.D., Tang, X.H.: Solutions on asymptotically periodic elliptic system with new conditions. Results Math. 70 539-565 (2016)

30. Qin, D.D., Tang, X.H., Wu, Q.F.: Ground states of nonlinear Schrödinger systems with periodic or non-periodic potentials. Commun. Pure Appl. Anal. 18(3), 1261-1280 (2019)

31. Sirakov, B:: On the existence of solutions of Hamiltonian elliptic systems in $\mathbb{R}^{N}$. Adv. Differ. Equ. 5, 1445-1464 (2000)

32. Struwe, M.: Variational Methods, Applications to Nonlinear Partial Differential Equations and Hamiltonian Systems. Springer, Berlin (2000)

33. Szulkin, A., Weth, T.: Ground state solutions for some indefinite variational problems. J. Funct. Anal. 257(12), 3802-3822 (2009)

34. Tang, X.H.: Non-Nehari manifold method for asymptotically linear Schrödinger equation. J. Aust. Math. Soc. 98 , 104-116 (2015)

35. Tang, X.H.: Ground state solutions of Nehari-Pankov type for a superlinear Hamiltonian elliptic system on $\mathbb{R}^{N}$. Can Math. Bull. 58, 651-663 (2015)

36. Wang, J., Xu, J.X., Zhang, F.B.: Existence of solutions for nonperiodic superquadratic Hamiltonian elliptic systems Nonlinear Anal. 72, 1949-1960 (2010)

37. Willem, M.: Minimax Theorems. Birkhäuser, Boston (1996)

38. Xia, L., Zhang, J., Zhao, F.K.: Ground state solutions for superlinear elliptic systems on $\mathbb{R}^{N}$. J. Math. Anal. Appl. 401 , 518-525 (2013)

39. Yang, J.: Nontrivial solutions of semilinear elliptic systems in $\mathbb{R}^{N}$. Electron. J. Differ. Equ. 6, 343-357 (2001)

40. Zhang, R.M., Chen, J., Zhao, F.K.: Multiple solutions for superlinear elliptic systems of Hamiltonian type. Discrete Contin. Dyn. Syst., Ser. A 30, 1249-1262 (2011)

41. Zhang, J., Qin, W.P., Zhao, F.K.: Existence and multiplicity of solutions for asymptotically linear nonperiodic Hamiltonian elliptic system. J. Math. Anal. Appl. 399, 433-441 (2013)

42. Zhang, W., Zhang, J., Tang, X.H.: Multiple solutions for asymptotically quadratic and superquadratic elliptic system of Hamiltonian type. Appl. Math. Comput. 263, 36-46 (2015)

43. Zhang, W., Zhang, J., Mi, H.: On fractional Schrödinger equation with periodic and asymptotically periodic conditions Comput. Math. Appl. 74, 1321-1332 (2017)

44. Zhang, J., Zhang, W., Tang, X.H.: Ground state solutions for Hamiltonian elliptic system with inverse square potential. Discrete Contin. Dyn. Syst. 37, 4565-4583 (2017)

45. Zhao, F.K., Zhao, L.G., Ding, Y.H.: Multiple solutions for asymptotically linear elliptic systems. Nonlinear Differ. Equ. Appl. 15, 673-688 (2008)

46. Zhao, F.K., Zhao, L.G., Ding, Y.H.: Infinitely many solutions for asymptotically linear periodic Hamiltonian elliptic systems. ESAIM Control Optim. Calc. Var. 16, 77-91 (2010)

47. Zhao, F.K., Zhao, L.G., Ding, Y.H.: Multiple solutions for a superlinear and periodic elliptic system on $\mathbb{R}^{N}$. Z. Angew. Math. Phys. 62, 495-511(2011)

48. Zhao, L.G., Zhao, F.K.: On ground state solutions for superlinear Hamiltonian elliptic systems. Z. Angew. Math. Phys. 64, 403-418 (2013) 